\title{
EPS 9: TRENDS IN PHYSICS
}

9th General Conference of the EUROPEAN PHYSICAL SOCIETY

Palazzo dei Congressi, Florence, Italy, 14-17 September 1992

In conjunction with:

- Celebration of the 25th Anniversary of EPS

- General Meeting of EPS Ordinary Members

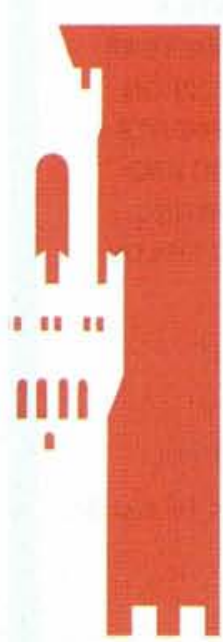

European Quantum Electronics Conference (10-13 September)

- 2nd EPS Liquid Matter Conference (18-22 September)

R.A. Ricci (on the left), EPS 9 Chairman, and

E. Brézin, Programme Committee Chairman.

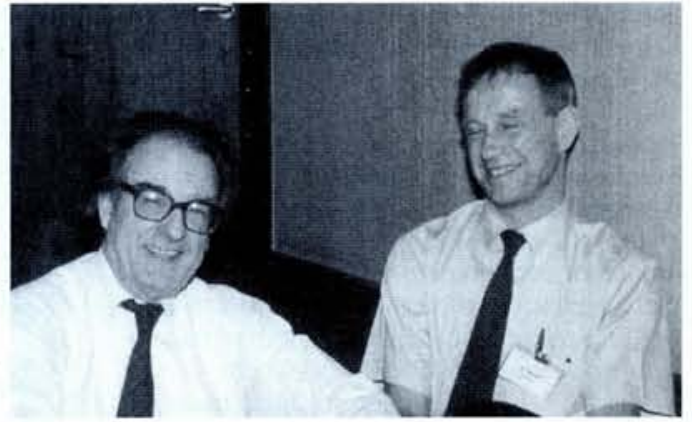

Contributed Papers and Posters

Contributions are invited on topics related to those covered by the parallel symposia. They will mainly be presented as posters, but a few will be selected before the conference by the symposia organizers for oral presentation (authors must indicate if they do not wish to be considered for an oral presentation). Each registered author is entitled to make only one contribution. One hour each day will be devoted to the poster sessions.

Camera-ready abstracts by 2 April 1992 to: Prof. A.M. Cartacci, Physics Department, Florence Univ., Largo E. Fermi, 2, I-50125 Florence.

\section{Registration}

Early registration by 15 May 1993 advised.

Address: OIC-MP, Via La Marmora, 24, I-50121 Florence.

The registration fees (including abstracts booklet and proceedings) in Italian lire are: - IOM's: 260000.- / Students: 50000.- / NonMembers: $380000 .-/$ Members of National or Collaborating Societies: 300000 .-

For student grants (which are limited in number), contact:

Prof. E. Bogani, Physics Dept., Florence Univ., Largo E. Fermi, 2, I-50125 Florence.
Plenary lectures:

W. Buckel, Karlsruhe

F.T. Arecchi, Florence

G. Charpak, Geneva

C. Cohen-Tannoudji, Paris

P.G. de Gennes, Paris

J. Ellis, Geneva

Y. Imry, Rehovot

W. Krätschmer, Heidelberg

S.B. Luitjens, Eindhoven

J. Mason, London

A. Pais, New York

G. Parisi, Rome

M.J. Rees, Cambridge

H. Rohrer, Zurich

C. Rubbia, Geneva

P.J. Twin, Liverpool

D.J. Wallace, Edinburgh

P. Wyder, Grenoble

On the responsibility of scientists to the public (Powell Memorial) Space-time chaos and topological defects in optics

From detector physics to medical applications

Laser cooling and trapping of atoms

4000 years of polymer research

Known and unknown matter

Mesoscopic disordered systems

Fullerenes and fullerites: new forms of carbon

Develoments in storage density of magnetic optical recording

The greenhouse effect and global warming

History of physics

Immunological networks

The emergence of structure of the universe

The dawn of nanometrage

Experimental particle physics (title to be announced)

Superdeformed nuclei

Physics with supercomputers

Physics in high magnetic fields

Parallel symposia (and organizers' names):

History of physics: F. Bevilacqua, Pavia

Local probes - STM: U. Dürig, Rüschlikon

Liguid-glass transition: T. Geszti, Budapest

Magnetic multilayers: P. Gruenberg, Jülich

Fluid surfaces: W. Helfrich, Berlin

Heavy ions: H.J. Specht, Heidelberg

Physics with synchrotron radiation:

M. Altarelli, Grenoble

Quantum wells and superconductors:

$$
\text { G. Bastard, Paris }
$$

Standard Model and beyond:

C. Kounnas, Geneva

Optics and quantum mechanics:

D. Lenstra, Amsterdam

Inertial fusion: R. Bock, Darmstadt

Femtosecond spectroscopy: G. Geber, Freiburg

Exotic nuclei: P. Gregers-Hansen, Aarhus

Non-ideal plasmas: W.D. Kräft, Griefswald

Flux physics: P.H. Kes, Leiden

Quantum fluids: S. Stringari, Trento

Optics and interferometry with atoms: A. Aspect, Orsay

Accelerators, storage rings: M. Promé, Saclay

Soft matters - polymers, liquid cystals, aerogels: P. Pieranski, Orsay

Physics in a changing world:

E.W.A. Lingeman, Amsterdam

Interplay between particle physics and astrophysics: E. Belotti, Milan

Low-dimensional systems and mesoscopic systems: P.C. Main, Nottingham

Statistical mechanics - rigorous results, 2D systems: M. Rasetti, Turin

Collisions of multiply charged ions with surface and gases: R. Morgenstern, Göttingen

\section{Dusty Disk Fuels a Possible Black Hole}

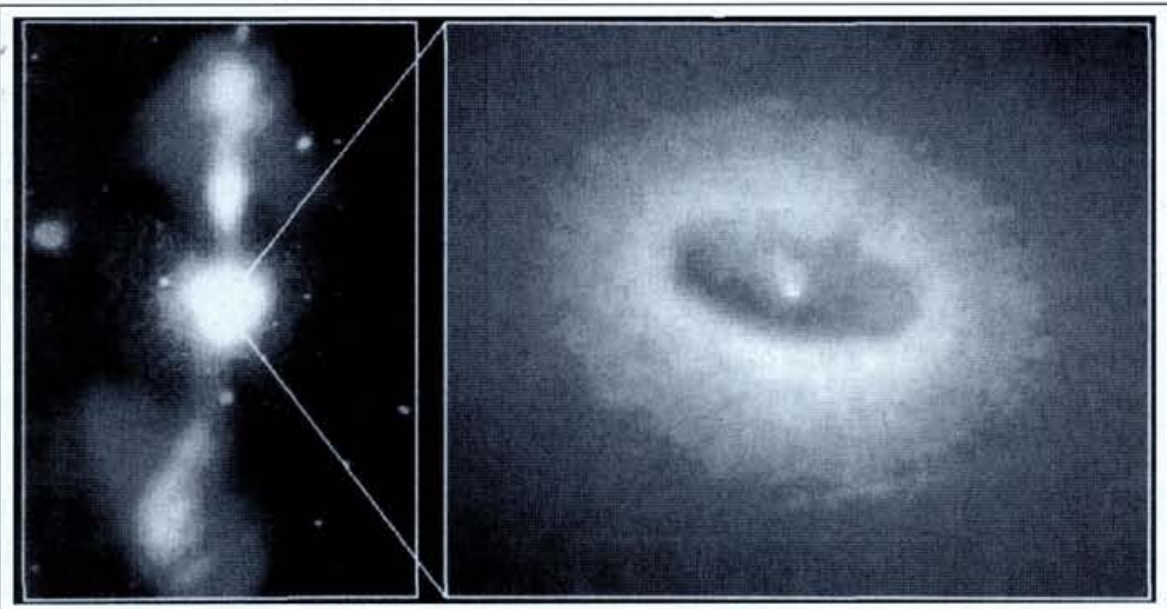

Astronomers using the Hubble Space Telescope's Wide Field/Planetary Camera reported on 19 November that they had observed what appears to be a black hole at the centre of an active galaxy (NGC 4261 in the Virgo Cluster). The 400 light-years in diameter dark disk shown in the Hubble image on the right is the cold, core region which extends to within a few hundred million miles of the suspected black hole. Matter fed into the black hole by the disk is heated owing to compression by gravity and hot gas is squirted out as jets. The jets show up in the composite optical/radio image on the left taken by the Observatory del Roque de los Muchachos, Canary Islands. The central bright region of the composite-image indicates that the 30000 light-years in diameter galaxy is unremarkable.

There remain some mysteries of course. For instance, the disk is believed to be composed of dust, but where does the dust come from? One possibility is that it is a remnant of a spiral galaxy which was swallowed up by NGC 4261. Spectroscopy of the disk is planned after the Shuttle repair mission in late1993 , as in the words of $\mathrm{H}$. Ford, a co-investigator on the mainly US team, "this is the first case where we can follow the disk's gas in an orderly way down to the immediate environment of a black hole". 